\title{
LBM simulation of interfacial behaviour of bubbles flow at low Reynolds number in a square microchannel
}

\author{
Y. Y. Yan \& Y. Q. Zu \\ School of the Built Environment, University of Nottingham, UK
}

\begin{abstract}
Bubbles flow in a microchannel is an interfacial and surface tension dominated problem. The paper reports results of numerical modelling and simulation of interfacial behaviour of bubbles flow and coalescence in a square microchannel. The lattice Boltzmann method (LBM) is developed and applied to the simulation in which a simple linear function is applied to the order parameter to approximate the density within the interface of gas-liquid. The evolution of two isothermal air bubbles flowing through a water-filled microchannel at low Reynolds number and the interactions between the flow fields and the interface of gas-liquid are simulated and investigated numerically.
\end{abstract}

Keywords: bubbles, coalescence, microchannel, LBM, interfacial behaviour.

\section{Introduction}

To understand bubbles interfacial behaviour in two-phase flow has long been an important topic of research in physical science and engineering; it becomes particular important for studying the flow in a confined system such as microchannel. Over the past few years, the study of gas-liquid two-phase flow in microchannels has drawn much attention from scientists and engineers due to increasing demands for developing micro-fluidic devices, micro-heatexchangers, micro-reactors, micro-actuators, etc.

On experimental study, in the last decades, many results on adiabatic gasliquid two-phase flow patterns in micro/mini-channels were reported [1-3], but they poorly agree with the previous transition models and correlations for macrochannels. Chen et al. [4] tested nitrogen-water two-phase flow in circular mini/micro channels to report flow patterns including bubbly, slug, bubble-train 
slug, churn and annular flows. Other experimental studies of gas-water flow in microchannels can also be identified [5-9].

On numerical study, in recent years, along with extensive applications of CFD to the study of two-phase flow [10-13], the lattice Boltzmann method (LBM) has become an established numerical scheme for simulating multiphase fluid flows. The key idea behind the LBM is to recover correct macroscopic motion of fluids by incorporating the complicated physics of problems into simplified microscopic models or mesoscopic kinetic equations. In LBM, kinetic equations of particle velocity distribution functions are first solved; macroscopic quantities are then obtained by evaluating hydrodynamic moments of the distribution function. This intrinsic feature enables the LBM to model phase segregation and interfacial dynamics of multiphase flow. Therefore, the LBM has a potential and broad applicability as well as many computational advantages such as parallel of algorithm and simplicity of programming [14-15]. Since the last 20 years, different LBM models for simulating multiphase flow have been developed. Gunstensen et al. [16] proposed a multi-component model based on the two-component lattice gas method. Shan and Chen [17] presented a twophase/component flow model of mean-field interactions. Later, Swift et al. [18, 19] proposed a free energy model; and He et al. [20] developed the model using index function to track the interface of multi-phase flow with large density ratios. In 2004, Inamuro et al. [21] developed a LBM model based on the projection method to predict the behaviours of incompressible bubbles/particles in bulk liquid. The method calculates two distribution functions of particle velocity to track the interface and to predict velocities; the corrected velocity field satisfying the continuity equation is obtained by solving the Poisson equation. Recently, a further work of the LBM for incompressible two-phase flow on a partial wetting surface with large density ratio was presented by the author [22].

In the present paper, two isothermal air bubbles motion and coalescence in a water-filled rectangular microchannel are studied to test the suitability of the LBM for simulating the interfacial behaviour of the bubbles flow in a micro channel.

\section{Methodology}

\subsection{The lattice Boltzmann method}

A new scheme of the lattice Boltzmann method for simulating two-phase fluid of large density ratio is proposed and described below. In a 3-dimensional 15velocity (D3Q15) LBM model, as shown in Fig. 1, the particle velocity, $\mathbf{e}_{\alpha}(\alpha=0,1, \ldots, 14)$, is given by

$$
\begin{aligned}
& {\left[\mathbf{e}_{0}, \mathbf{e}_{1}, \mathbf{e}_{2}, \mathbf{e}_{3}, \mathbf{e}_{4}, \mathbf{e}_{5}, \mathbf{e}_{6}, \mathbf{e}_{7}, \mathbf{e}_{8}, \mathbf{e}_{9}, \mathbf{e}_{10}, \mathbf{e}_{11}, \mathbf{e}_{12}, \mathbf{e}_{13}, \mathbf{e}_{14}\right]} \\
& =\left[\begin{array}{cccccccccccccccc}
0 & 1 & 0 & 0 & -1 & 0 & 0 & 1 & -1 & 1 & 1 & -1 & 1 & -1 & -1 \\
0 & 0 & 1 & 0 & 0 & -1 & 0 & 1 & 1 & -1 & 1 & -1 & -1 & 1 & -1 \\
0 & 0 & 0 & 1 & 0 & 0 & -1 & 1 & 1 & 1 & -1 & -1 & -1 & -1 & 1
\end{array}\right] .
\end{aligned}
$$



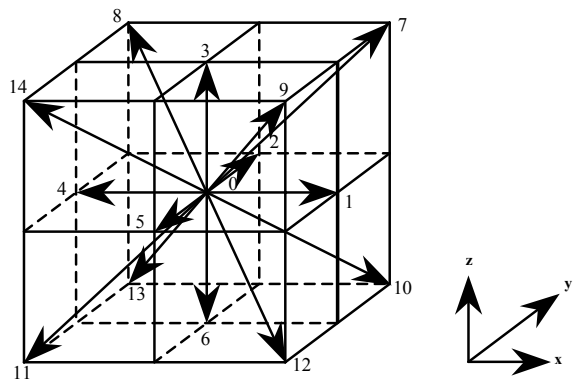

Figure 1: Discrete velocity set of 3-D 15-velocity (D3Q15) model.

To simulate two-phase fluid flow, two distribution functions of fluid particle velocity, $f_{\alpha}$ and $g_{\alpha}$, are introduced. Function $f_{\alpha}$ is used to calculate the order parameter $\phi$, which distinguishes the two phases. Function $g_{\alpha}$ is used to calculate the predicted velocity, $\mathbf{u}^{*}$, of the two-phase fluids. The evolution of the particle distribution functions $f_{\alpha}(\mathbf{x}, t)$ and $g_{\alpha}(\mathbf{x}, t)$ with particle velocity $\mathbf{e}_{\alpha}$ at point $\mathbf{x}$ and time $t$ is calculated by the following equations:

$$
\begin{aligned}
& f_{\alpha}\left(\mathbf{x}+\mathbf{e}_{\alpha} \delta_{t}, t+\delta_{t}\right)=f_{\alpha}^{(e q)}(\mathbf{x}, t) ; \\
& g_{\alpha}\left(\mathbf{x}+\mathbf{e}_{\alpha} \delta_{t}, t+\delta_{t}\right)=g_{\alpha}^{(e q)}(\mathbf{x}, t) ;
\end{aligned}
$$

where, $\delta_{t}=1$ is the time step in which the particles travel the lattice spacing; $f_{\alpha}^{(e q)}$ and $g_{\alpha}^{(e q)}$ are the corresponding equilibrium states of $f_{\alpha}$ and $g_{\alpha}$, given by

$$
\begin{gathered}
f_{\alpha}^{(e q)}(\mathbf{x}, t)=H_{\alpha} \phi+F_{\alpha}\left[p_{0}-k \phi \nabla^{2} \phi-\frac{k}{6}|\nabla \phi|^{2}\right]+3 \omega_{\alpha} \phi\left(\mathbf{e}_{\alpha}^{\prime} \cdot \mathbf{u}\right) ; \\
+\omega_{\alpha} k \mathbf{e}_{\alpha}^{\prime} \cdot \mathbf{G}(\phi) \cdot \mathbf{e}_{\alpha} \\
g_{\alpha}^{(e q)}(\mathbf{x}, t)=\omega_{\alpha}\left[1+3\left(\mathbf{e}_{\alpha}^{\prime} \cdot \mathbf{u}\right)+\frac{9}{2}\left(\mathbf{e}_{\alpha}^{\prime} \cdot \mathbf{u}\right)^{2}-\frac{3}{2} \mathbf{u}^{2}+\frac{3}{4} \mathbf{e}_{\alpha}^{\prime} \cdot(\nabla \mathbf{u}+\mathbf{u} \nabla) \cdot \mathbf{e}_{\alpha}\right] \\
+\omega_{\alpha} \frac{k}{\rho} \mathbf{e}_{\alpha}^{\prime} \cdot \mathbf{G}(\phi) \cdot \mathbf{e}_{\alpha}-\frac{2}{3} F_{\alpha} \frac{k}{\rho}|\nabla \phi|^{2}+3 \omega_{\alpha} \frac{1}{\rho} \nabla \cdot[\mu(\nabla \mathbf{u}+\mathbf{u} \nabla)] \cdot \mathbf{e}_{\alpha}
\end{gathered}
$$

where,

$$
\omega_{\alpha}=\left\{\begin{array}{ll}
2 / 9, & \alpha=0 \\
1 / 9, & \alpha=1, \ldots, 6 \\
1 / 72, & \alpha=7, \ldots, 14
\end{array}, F_{\alpha}=\left\{\begin{array}{ll}
-7 / 3, & \alpha=0 \\
1 / 3, & \alpha=1, \ldots, 6 \\
1 / 24, & \alpha=7, \ldots, 14
\end{array}, H_{\alpha}=\left\{\begin{array}{l}
1, \alpha=0 \\
0, \alpha=1, \ldots, 14
\end{array} ;\right.\right.\right.
$$

and

$$
\mathbf{G}(\phi)=\frac{9}{2}(\nabla \phi)(\phi \nabla)-\frac{3}{2}|\nabla \phi|^{2} \mathbf{I}
$$

In the above equations, $k$ is a constant parameter for determining the width of interface and the strength of surface tension; $I$ is a unit tensor of secondorder. Given that $\psi(\phi)$ is the bulk free-energy density, then 


$$
p_{0}=\phi \frac{\partial \psi}{\partial \phi}-\psi
$$

The macroscopic quantities, $\mathbf{u}^{*}, \phi, \rho, \mu$ can be evaluated as

$$
\begin{gathered}
\phi=\sum_{\alpha} f_{\alpha}, \quad \mathbf{u}^{*}=\sum_{\alpha} \mathbf{e}_{\alpha} g_{\alpha}, \\
\rho= \begin{cases}\rho_{G}, & \phi<\phi_{G} \\
\frac{\phi-\phi_{G}}{\phi_{L}-\phi_{G}}\left(\rho_{L}-\rho_{G}\right)+\rho_{G}, & \phi_{G} \leq \phi \leq \phi_{L}, \\
\rho_{L}, & \phi>\phi_{L}\end{cases} \\
\mu=\frac{\rho-\rho_{G}}{\rho_{L}-\rho_{G}}\left(\mu_{L}-\mu_{G}\right)+\mu_{G},
\end{gathered}
$$

where $\phi_{L}$ and $\phi_{G}$ are respectively the maximum and minimum order parameter for marking bulk liquid and gas; $\rho_{L}$ and $\rho_{G}$ are respectively the density of liquid and gas phases; $\mu_{L}$ and $\mu_{G}$ are respectively the dynamic viscosity of liquid and gas phases. In Eq. (10), a simple linear function is applied to approximate the density within the interface; this enables the present method to obtain $f_{\alpha}^{(e q)}(\mathbf{x}, t)$ and $g_{\alpha}^{(e q)}(\mathbf{x}, t)$ in a simple form and thereby improve computation efficiency. For example, the calculations of second-order tensor $\mathbf{G}(\rho)$, first partial derivative of $\rho$, etc., can be avoided in the present model, but they have to be calculated in the models such as [21].

To enable the method to treat two-phase fluids interacting with confined solid surfaces with wetting boundary potentials, for the current isothermal system, a simple form of representation of the free energy density $\psi(\phi)$, as suggested in [23], rather than the van der Waals free energy used in the traditional model, is applied in the present simulation, namely,

$$
\psi(\phi)=\beta\left(\phi-\phi_{G}\right)^{2}\left(\phi-\phi_{L}\right)^{2}+\mu_{b} \phi-p_{b} ;
$$

where $\beta$ is a constant relating to interfacial thickness; $\mu_{b}$ and $p_{b}$ are the bulk chemical potential and bulk pressure, respectively.

By substitution of Eq. (11), Eq. (7) becomes

$$
p_{0}=\beta\left(\phi-\phi_{L}\right)\left(\phi-\phi_{G}\right)\left(3 \phi^{2}-\phi \phi_{L}-\phi \phi_{G}-\phi_{L} \phi_{G}\right)+p_{b} .
$$

In a plane interface under an equilibrium condition, the density profile across the interface is on equilibrium and can be represented as [22]

$$
\phi(\xi)=\frac{\phi_{L}+\phi_{G}}{2}+\frac{\phi_{L}-\phi_{G}}{2} \tanh \left(\frac{2 \xi}{D}\right) ;
$$

where $\xi$ is the coordinate normal to the interface; the interface thickness $D$ is given by

$$
D=\frac{4}{\phi_{L}-\phi_{G}} \sqrt{\frac{k}{2 \beta}} .
$$


The fluid-fluid (liquid-gas) surface tension force $\sigma$ is expressed as [24]

$$
\sigma=\frac{\left(\phi_{L}-\phi_{G}\right)^{3}}{6} \sqrt{2 k \beta}
$$

\subsection{Correction for pressure}

It should be pointed out that the predicted velocity $\mathbf{u}^{*}$ is not divergence free. To obtain the velocity field which satisfies the continuity equation $(\nabla \cdot \mathbf{u}=0), \mathbf{u} *$ is corrected by following equations:

$$
\begin{gathered}
\mathbf{u}-\mathbf{u}^{*}=-\frac{\nabla p}{\rho}, \\
\nabla \cdot \mathbf{u}^{*}=\nabla \cdot\left(\frac{\nabla p}{\rho}\right) ;
\end{gathered}
$$

where $p$ is the pressure of the two-phase fluid. Eq. (17) can be approximated by the LBM framework equation:

$$
h_{\alpha}\left(\mathbf{x}+\mathbf{e}_{\alpha}, n+1\right)=h_{\alpha}(\mathbf{x}, n)-\frac{1}{\tau}\left[h_{\alpha}(\mathbf{x}, n)-\omega_{\alpha} p(\mathbf{x}, n)\right]-\frac{\omega_{\alpha}}{3} \frac{1}{\rho} \nabla \cdot \mathbf{u} * ;
$$

where, $n$ is the number of iterations and $\tau=0.5+1 / \rho$ is the relaxation time. The pressure at step $n+1$ is given by

$$
p(\mathbf{x}, n+1)=\sum_{\alpha} h_{\alpha}(\mathbf{x}, n+1) .
$$

The convergent pressure $p$ is determined when

$$
\forall \mathbf{x} \in V,|p(\mathbf{x}, n+1)-p(\mathbf{x}, n+1)|<\varepsilon ;
$$

where $V$ denotes the whole computational domain. Substituting the newly obtained pressure $p$ into and solving Eq. (16) gives the corrected $\mathbf{u}$, the velocity field.

\subsection{Boundary treatment}

Applying the present LBM model, no-slip boundary conditions can be implemented by simply specifying the zero velocity on the solid boundaries, i.e. the boundary velocities $\mathbf{u}$ and $\mathbf{u} *$ in Eqs. (4-5) and (16-18) are given by

$$
\mathbf{u}_{w}=0, \mathbf{u}_{w}^{*}=0 .
$$

As there is always a thin liquid layer in the vicinity of the solid boundary surface due to the intermolecular forces between the liquid and solid substrate $[25,26]$, it is assumed that a thin liquid occupies one layer of the lattice spacing, the order parameter on the boundary used in Eqs. (4-5) is then determined by

$$
\phi_{w}=\phi_{L} \text {. }
$$

In the present simulation, the finite-difference of the order parameter on the boundary are given by

$$
\left.\frac{\partial^{2} \phi}{\partial \zeta^{2}}\right|_{\zeta=0} \approx \frac{1}{2}\left(-\left.3 \frac{\partial \phi}{\partial \zeta}\right|_{\zeta=0}+\left.4 \frac{\partial \phi}{\partial \zeta}\right|_{\zeta=1}-\left.\frac{\partial \phi}{\partial \zeta}\right|_{\zeta=2}\right)
$$


where, $\zeta$ is the direction perpendicular to the wall. In this scheme, the first term on the right hand side of Eq. (23) is determined by a right-handed finitedifference; the second term is calculated by a standard centred finite-difference formula. Finally, it is found empirically that the best choice for the third term is a left-handed finite-difference formula taken back into the wall, namely,

$$
\left.\frac{\partial \phi}{\partial \zeta}\right|_{z=2} \approx \frac{1}{2}\left(\left.3 \phi\right|_{\zeta=2}-\left.4 \phi\right|_{\zeta=1}+\left.\phi\right|_{\zeta=0}\right)
$$

\section{Results and discussion}

The motion of air bubbles surrounded by water flow in a three dimensional rectangular microchannel is considered. The gravitational force is taken into account by adding the term $-3 \omega_{\alpha} \mathbf{e}_{\alpha z}\left(1-\rho_{G} / \rho\right) g$ to the right hand side of Eq. (3), where $g$ is the dimensionless gravitational acceleration.

Fig. 3 shows the computational domain and initial and boundary conditions of the modelling. Initially, two air bubbles with same diameter $d=200 \mu \mathrm{m}$ are placed $300 \mu m$ apart in water inside the channel of the length $\widetilde{L}_{x}=1200 \mu m$, the width and the height $\widetilde{L}_{y}=\widetilde{L}_{z}=300 \mu m$. The channel has an inlet boundary on the left hand side of the channel and a free outflow boundary on the right hand side of the channel. The other four sides of the channel are no-slip solid walls. Naturally, the densities of two fluids are set at $\tilde{\rho}_{L}=1000 \mathrm{~kg} / \mathrm{m}^{3}$ and $\tilde{\rho}_{G}=1.0 \mathrm{~kg} / \mathrm{m}^{3}$ (making the density ratio to be 1000); meanwhile the dynamic viscosities of them are $\tilde{\mu}_{L}=1 \times 10^{-3} \mathrm{~kg} / \mathrm{ms}, \quad \tilde{\mu}_{G}=2.1 \times 10^{-5} \mathrm{~kg} / \mathrm{ms}$, respectively. The initial surface tension between water and air is of $\tilde{\sigma}=1 \times 10^{-3} \mathrm{~kg} / \mathrm{s}^{2}$ and the gravitational acceleration is at $\tilde{g}=9.8 \mathrm{~m} / \mathrm{s}^{2}$. To relate the physical parameters with simulation parameters, a length scale of $L_{0}=1 \times 10^{-5} \mathrm{~m}$, time scale of $T_{0}=1 \times 10^{-7} \mathrm{~s}$ and mass scale of $M_{0}=1 \times 10^{-14} \mathrm{~kg}$ are applied; these lead to the dimensionless parameters: $\rho_{L}=100 ; \rho_{G}=0.1$; $\mu_{L}=0.1 ; \mu_{G}=2.1 \times 10^{-3} ; \phi_{L}=0.4 ; \phi_{G}=0.1 ; k=0.05 ;$ and $g=9.8 \times 10^{-9}$, respectively. Unless otherwise specified, the following simulations are within a computational domain occupied by $120 \times 30 \times 30$ cubic lattices; $\varepsilon$ in Eq. (20) is set at $\varepsilon=1 \times 10^{-6}$.

The velocity distribution at the inlet boundary is specified as,

$$
\left\{\begin{array}{l}
u_{x}(0, y, z)=16 U\left(L_{y}-y\right)\left(L_{z}-z\right) y z /\left(L_{y} L_{z}\right)^{2} \\
u_{y}(0, y, z)=0 \\
u_{z}(0, y, z)=0
\end{array}\right.
$$




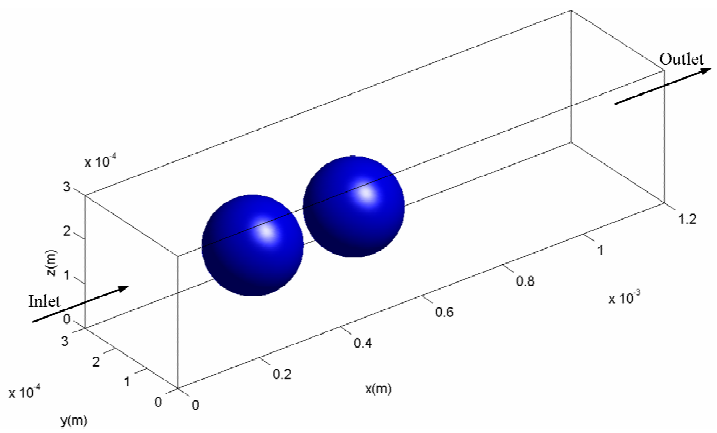

Figure 2: Computational domain and initial/boundary conditions.

where, $U$ is the maximum value of $u_{x}(0, y, z)$. Thus, the Reynolds number is defined as

$$
\operatorname{Re}=\frac{\rho_{L} U L_{z}}{\mu_{L}} .
$$

The bubbles flow and behaviour in the microchannel at $\mathrm{Re}=100$ is first simulated. The evolution with time of bubbles shapes and the behaviour of interactions are shown in Fig. 4. It can be seen clearly that the bubbles move in $x$-direction by the thrust force of surrounding water flow and meanwhile go up in $y$-direction due to the effect of buoyancy force; and with time marching, the two bubbles coalesce into a lager one. To focus only on the shape evolution of the left bubble at the early stage, it is found that the lower part of the bubble moves more quickly in $x$-direction than the upper part, which is caused mainly by the effects of velocity boundary layer near the solid wall of the channel.

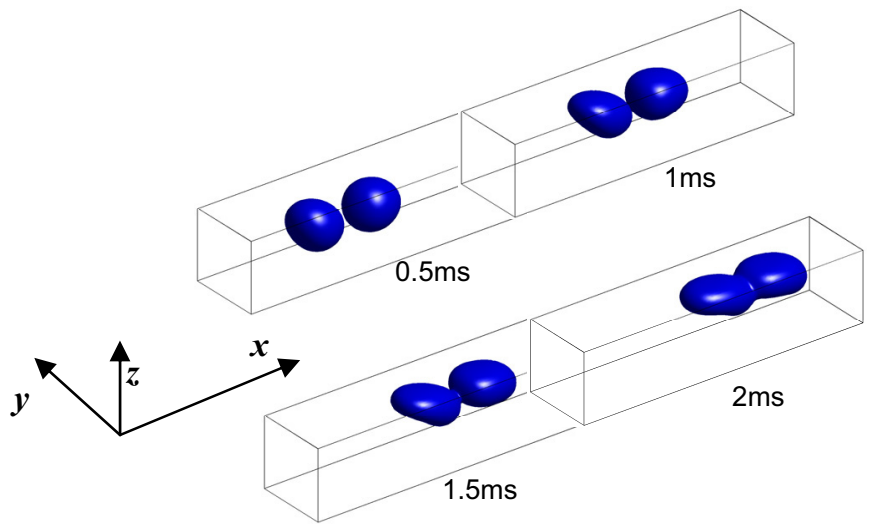

Figure 3: $\quad$ Evolution with time of bubble shapes and behaviour at $\mathrm{Re}=100$.

The velocity fields are obtained through the numerical modelling. For example, at $\tilde{t}=2 \times 10^{-3} \mathrm{~s}$, the velocity distribution at different cross sections of 
$y-z$ plane in Fig. 4 such as $x=L_{x} / 2,2 L_{x} / 3$ and $3 L_{x} / 4$, respectively, is shown in Fig. 5; where the solid line, the constant density line, indicates the interface between the two phases. As both pressure and velocity distributions across the interface are normally excellent indicators of numerical stability for the LBM calculations [27], Figs. 5 and 6 have actually shown that the present LBM can be used to obtain reasonable and stable velocity fields. Indeed, similar to the conventional CFD, the numerical instabilities of the LBM for two-phase flow of large density ratios are mainly caused by spurious velocities and/or the large oscillation of the pressure distribution across the phase interface. However, in the present method, the velocity and pressure are both corrected by solving an additional Poisson equation after each collision-stream step. Such corrections are able to ensure the velocity to satisfy the continuity equation and smooth pressure distributions even across the interface, so that to ensure the numerical stability.

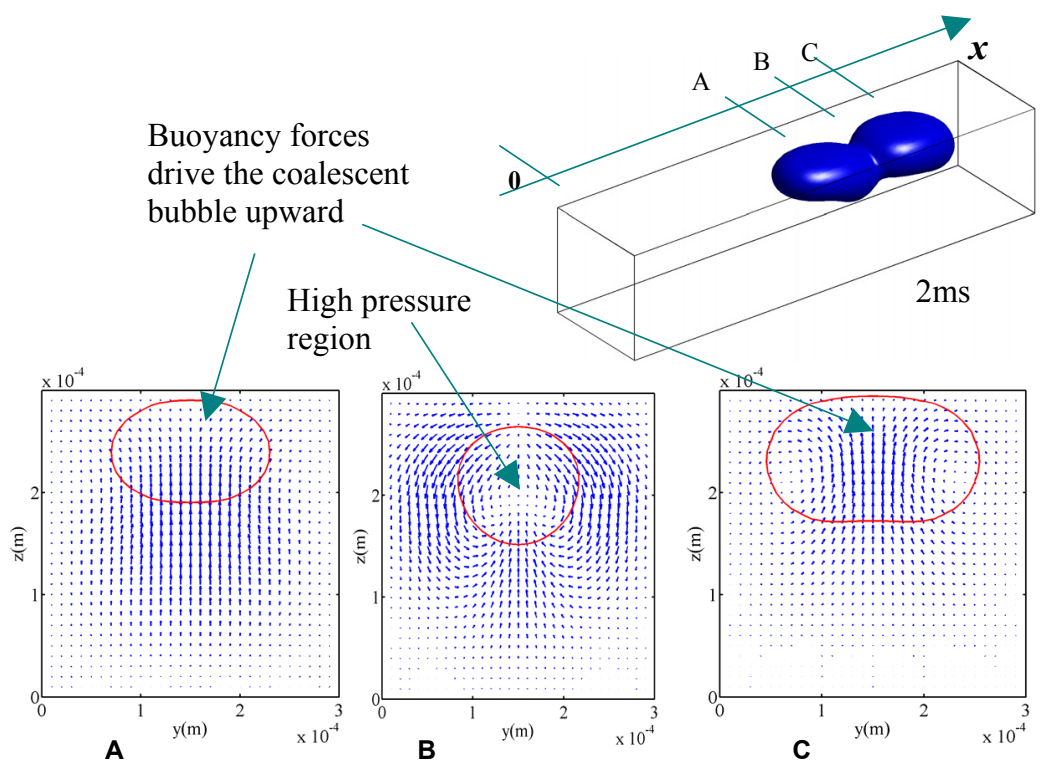

Figure 4: Velocity field at different cross section, $\tilde{t}=2 m s, \mathrm{Re}=100$ : (A: $\left.x=L_{x} / 2, \mathrm{~B}: x=2 L_{x} / 3, \mathrm{C}: x=3 L_{x} / 4\right)$.

Figs. 6(a) and (b) show the velocity vector and the vorticity contours, respectively, at $\tilde{t}=2 \times 10^{-3} s$, and at $y=L_{y} / 2$ on $x$-z plane. It can be seen that the local distribution in coherent structures is evident; the shape of the coalescent bubbles is a result of the interaction between the fields of velocity and density concentration, and this is mainly affected by the effects of buoyancy force of the bubbles [28].

Fig. 7 shows the evolution of the bubble shapes at a low Reynolds number $(\mathrm{Re}=50)$. The results show an interesting evolution of the bubbles flow and 

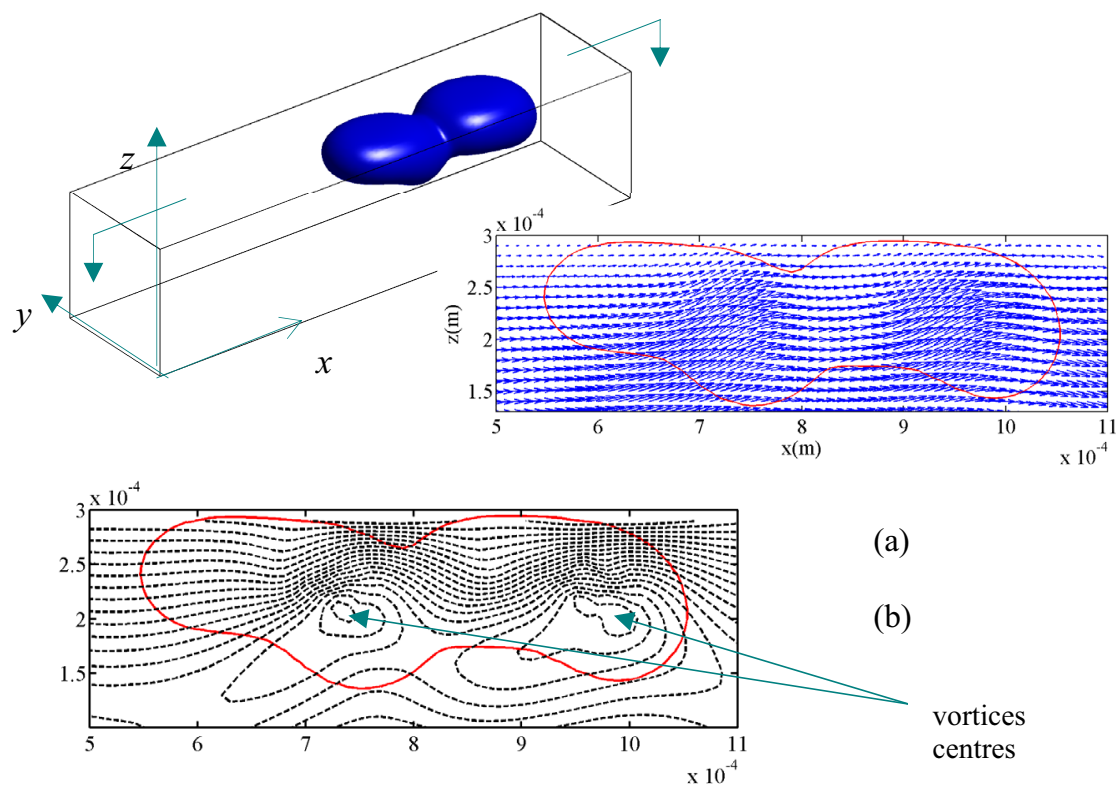

Figure 5: Velocity vector and vorticity contours of coalescent bubble at $y=L_{y} / 2$ on $\mathrm{X}-\mathrm{z}$ plane for $\tilde{t}=2 m s, \mathrm{Re}=100$.

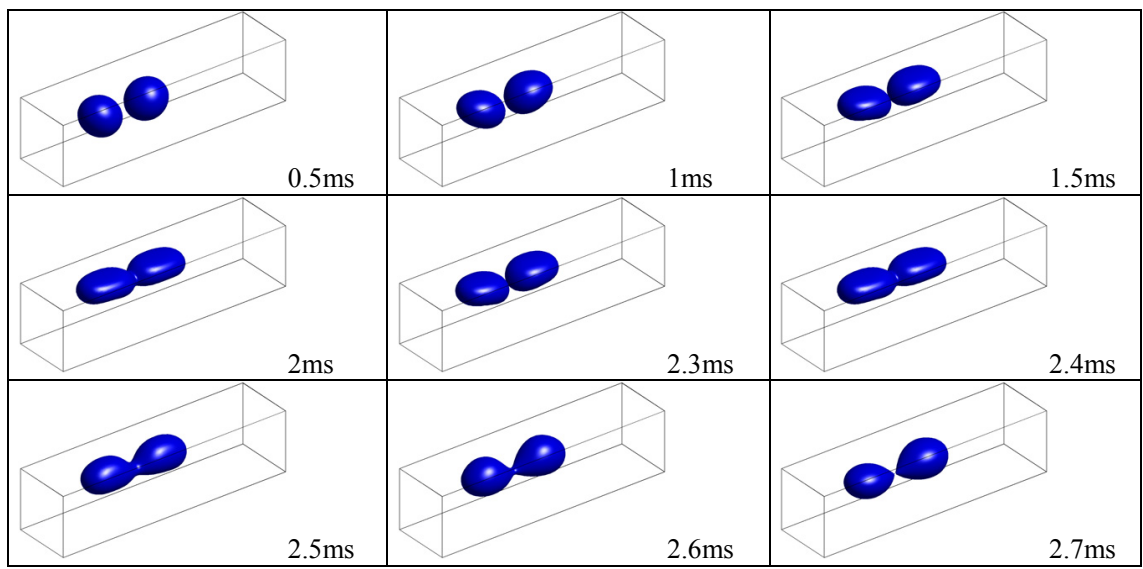

Figure 6: $\quad$ Time evolution of bubble shapes at $\mathrm{Re}=50$.

coalescence. At the early stage of the flow, the evolution of the bubbles is quite similar to that at $\mathrm{Re}=100$, two bubbles coalesce at $1.5 \sim 2.0 \mathrm{~ms}$; however, at the later stage, the newly coalescent bubble re-breaks up into two bubbles; this shows probably a typical phenomenon of the flow at low Reynolds number. 
Obviously, such separation is caused by the strong effect of shear boundary layer near the upstream boundary. As at low Reynolds number, the buoyancy effect is more evident; which forces the bubbles migrating to the upper boundary of the channel and meanwhile results in the displacements in $x$-direction smaller and forms a relative returning flow in the vicinity of the bubbles interface as the sheer stress tending to different directions.

\section{Conclusions}

In this paper, a newly modified LBM model is developed to simulate bubbles flow in a rectangular microchannel. In the current model, a simple linear function of order parameter is applied to approximate the density within the interface of two fluids; meanwhile, a new form of the free energy density (rather than the van der Waals free energy) is used to enable the model treat confined surface and wetting boundary conditions. Based on the developed LBM model, the evolution of two isothermal air bubbles move through a water-filled microchannel are investigated numerically. Both the bubble shapes and the velocity files are imported to analyze the bubble-water and bubble-bubble interactions. The effect of Reynolds number on the flow is also examined. It is found that two bubbles can finally coalesce into one larger bubble at the relatively high Reynolds number; however, under the lower Reynolds number (e.g. $\mathrm{Re}=50$ ), the newly coalesced bubble can be separated again by the stronger shear flow upstream.

\section{Acknowledgement}

This work is supported by the EPSRC under grant EP/D500125/1.

\section{References}

[1] Mishima, K., and Hibiki, T., 1996, Some characteristics of air-water twophase flow in small diameter vertical tubes, Int. J. Multiph. Flow, Vol. 22, pp 703-712.

[2] Coleman, J. W., and Garimella, S., 1999, Characterization of two-phase flow patterns in small diameter round and rectangular tubes, Int. J. Heat Mass Transf., Vol. 42, pp 2869-2881.

[3] Triplett, K. A., Ghiaasiaan, S. M., Abdel-Khalik, S. I., and Sadowski, D. L., 1999, Gas-liquid two-phase flow in microchannels - Part I: two-phase flow patterns, Int. J. Multiph. Flow, Vol. 25, pp 377-394.

[4] Chen, W. L., Twu, M. C., and Pan, C., 2002, Gas-liquid two-phase flow in micro-channels, Int. J. Multiph. Flow, Vol. 28, pp 1235-1247.

[5] Kawahara, A., Chung, P. M. Y., and Kawaji, M., 2002, Investigation of two-phase flow pattern, void fraction and pressure drop in a microchannel, Int. J. Multiph. Flow, Vol. 28, pp 1411-1435. 
[6] Chung, P. M. Y., Kawaji, M., Kawahara, A., and Shibata, Y., 2004, Twophase flow through square and circular microchannels - Effects of channel geometry, J. Fluids Eng., Trans. ASME, Vol. 126, pp 546-552.

[7] Cubaud, T., and Ho, C. M., 2004, Transport of bubbles in square microchannels, Phys. Fluids, Vol. 16, pp 4575-4585.

[8] Xiong, R. Q., and Chung, J. N., 2007, An experimental study of the size effect on adiabatic gas-liquid two-phase flow patterns and void fraction in microchannels, Phys. Fluids, Vol. 19, pp 1/033301-8/ 033301.

[9] Qu, W. L., Yoon, S. M., and Mudawar, I., 2004, Two-phase flow and heat transfer in rectangular micro-channels, J. Electron. Packag., Trans. ASME, Vol. 126, pp 288-300.

[10] Sussman, M., P. Smereka, S. Osher, A level set approach for computing solutions to incompressible two-phase flow, Journal of Computational Physics 114 (1994) 146-159.

[11] C.Y. Ji, Y.Y. Yan, 2008. A numerical study of bubbly flow in a rectangular microchannel. Proc. 6th Int. ASME Conf. on Nano-, Micro- and Minichannels, ICNMM2008-62117, June 23-25, 2008, Darmstadt.

[12] Y.Q. Zu, Y.Y. Yan, 2008, A numerical study of quasi-nucleate boiling in mini- and micro channels, ICNMM2008-62112, June 23-25, Darmstadt.

[13] Y.Q. Zu, S. Gedupudi, Y.Y. Yan, T.G. Karayiannis, D.B.R. Kenning, 2009, Numerical simulation and experimental observations of confined bubble growth during flow boiling in a mini-micro channel with a rectangular cross section of high aspect ratio, ICNMM2009-82118, Puhang, South Korea.

[14] Chen, Shiyi, and Doolen, Gary D., 1998, Lattice Boltzmann method for fluid flows, Ann. Rev. Fluid Mech., Vol. 30, pp 329-364.

[15] Succi, S., 2001, The lattice Boltzmann equation for fluid dynamics and beyond, Clarendon Press, Oxford.

[16] Gunstensen, A. K., Rothman, D. H., Zaleski, S., and Zanetti, G., 1991, Lattice Boltzmann Model of Immiscible Fluids, Phys. Rev. A, Vol. 43, pp 4320-4327.

[17] Shan, X. W., and Chen, H. D., 1993, Lattice Boltzmann Model for Simulating Flows with Multiple Phases and Components, Phys. Rev. E, Vol. 47, pp 1815-1819.

[18] Swift, M. R., Orlandini, E., Osborn, W. R., and Yeomans, J. M., 1996, Lattice Boltzmann simulations of liquid-gas and binary fluid systems, Phys. Rev. E, Vol. 54, pp 5041-5052.

[19] Swift, M. R., Osborn, W. R., and Yeomans, J. M., 1995, Lattice Boltzmann Simulation of Nonideal Fluids, Phys. Rev. Lett., Vol. 75, pp 830-833.

[20] He, X. Y., Chen, S. Y., and Zhang, R. Y., 1999, A lattice Boltzmann scheme for incompressible multiphase flow and its application in simulation of Rayleigh-Taylor instability, J. Comput. Phys., Vol. 152, pp 642-663.

[21] Inamuro, T., Ogata, T., Tajima, S., and Konishi, N., 2004, A lattice Boltzmann method for incompressible two-phase flows with large density differences, J. Comput. Phys., Vol. 198, pp 628-644. 
[22] Yan, Y.Y., Zu, Y.Q., 2007, A lattice Boltzmann method for incompressible two-phase flows on partial wetting surface with large density ratio. Journal of Computational Physics, 227(1), 763-775.

[23] Jamet, D., Lebaigue, O., Coutris, N., and Delhaye, J. M., 2001, The second gradient theory: a tool for the direct numerical simulation of liquid-vapor flows with phase-change, Nucl. Eng. Des., Vol. 204, pp 155-166.

[24] Rowlinson, J.S., and Widom, B., 1989, Molecular Theory of Capillarity, Clarendon, Oxford.

[25] Wayner, P.C., 1999, Intermolecular forces in phase-change heat transfer: 1998 Kern Award Review, AIChE Journal, 45(10), 2055-2068.

[26] Ji, C., Yan, Y.Y., 2008, A molecular dynamics simulation of liquid-vapoursolid system near triple-phase contact line of flow boiling in a microchannel. Applied Thermal Engineering, 28(2-3), 195-202.

[27] Lee, T., and Lin, C. L., 2005, A stable discretization of the lattice Boltzmann equation for simulation of incompressible two-phase flows at high density ratio, J. Comput. Phys., Vol. 206, pp 16-47.

[28] Mazzitelli, I. M., Lohse, D., and Toschi, F., 2003, The effect of microbubbles on developed turbulence, Phys. Fluids, Vol. 15, pp L5-L8. 